\title{
A model of a production-repair inventory system with time-varying demand and quality- dependent recovery channels
}

\author{
Ivan $\mathrm{Yeo}^{1 *}$ \\ ${ }^{1}$ Centre for Mathematical Sciences, Universiti Tunku Abdul Rahman, Malaysia
}

\begin{abstract}
In this paper, we study an inventory system over an infinite planning horizon where a time-varying demand is satisfied by process cycles that consist of a production batch followed by a recovery batch. Our model considers three types of inventory-returned items, serviceable items, and raw material. Furthermore, our model considers two recovery channels - recovery into serviceable items and recovery into raw material. Serviceable items are thus sourced from two inputs-direct recovery and production from raw material. These raw materials can be salvaged from returned items, as well as bought from external sources whenever required. We propose an expression for the unit time total cost as well as a numerical method to find the optimal policy. The properties of the model are studied through numerical experiments, in particular, the feasible situations where hybrid policies are better than pure policies.
\end{abstract}

\section{Introduction}

Reuse is a phenomenon that has been around for some time but is now receiving increasing attention due to the growth of environmental concerns. Reuse of metal scrap, waste paper, and soft drink bottles are some of the more ubiquitous examples. In these cases, recovery of the used items is more appealing from an economic point of view than disposal [1]. In addition to economic benefits, reuse slows down the growth of landfills and the accumulation of noxious solid waste in the environment. Reuse of old items often consumes less resources than producing brand new items, and one salient result is lower carbon emissions. Today, the environmental costs that are incurred during a product's life cycle are starting to play a more important role in the calculation of the product's total production cost [2].

The study of inventory models with reuse began with authors modifying the classical economic order quantity (EOQ) model by Harris [3]. As far as we know, Schrady [4] was the first author to propose an EOQ model for an inventory system with reuse. $\mathrm{He}$ considered a single-item system where demand is satisfied by both the procurement of new items and the repair of returned items. He assumed that procurement and repair have infinite rates, and all returned items are reparable. He considered the policies in which one

*Corresponding author: yeohg@utar.edu.my 
procurement setup is alternated with $R$ repair setups (or $(1, R)$ policies) and derived EOQ formulas for these policies.

Nahmias and Rivera [5] extended the work of Schrady [4] to the case where the repair rate is finite. They derived EOQ formulas for $(1, R)$ policies as well. Later, Mabini et al. [6] also extended the work of Schrady [4] by considering a multi-item system, where different types of items use the same repair facility, and where shortages are allowed to occur. They proposed numerical solution procedures instead of EOQ formulas.

Later, Koh et al. [7] considered two types of policies: $(1, R)$ policies and $(P, 1)$ policies - a $(P, 1)$ policy alternates one recovery setup with $P$ production setups. They considered two cases for each policy: the case where the repair rate exceeds the demand rate and the case where the repair rate is equal to or is less than the demand rate. They also assumed finite repair rate and infinite production rate. They derived EOQ formulas and found the optimal integer setup numbers $R$ and $P$ through a numerical search procedure. In a follow up work, Konstantaras and Papachristos [8] obtained closed-form expressions for these optimal integer setup numbers.

Teunter [9] generalized the works of his predecessors by considering the case of finite production rate and recovery rate for $(1, R)$ and $(P, 1)$ policies. He simplified the resultant integer programming problem by treating the setup numbers $R$ and $P$ as real values, and using the closest positive integers in his solution. His algorithm gave an approximate optimal policy, but he was able to show numerically that his results are relatively good in most cases. In two follow up works $[10,11]$, the authors proposed exact solution algorithms.

The above authors assumed that all returned items are reusable. Richter relaxed this assumption by proposing two EOQ waste disposal models $[12,13]$, where a portion of the returned items are not reused but are scrapped at a cost. Richter assumed that the production and repair rates are infinite, and one production setup alternates with one repair setup during each cycle [12], or $n$ production setups occur successively followed by $m$ repair setups during each cycle [13]. Later, Richter [14] and Dobos [15] extended the works in $[12,13]$ by considering the problem of finding the optimal setup numbers as an integer programming problem. In another extension, Teunter [16] proposed an EOQ waste disposal model where repaired items and manufactured items that serve the same demand process have different holding costs. Later, Dobos [17] considered finite production rate and repair rate for the single production setup and a single repair setup per cycle policy (or $(1,1)$ policy). Finally, Dobos [18] proposed a general model with $P$ production setups and $R$ repair setups in a cycle (or $(P, R)$ policy).

Mokhtari [19] proposed an EPQ (economic production quantity) model in which demand is satisfied by items that are reusable for a finite number of times instead of just once, and he derived formulas for the optimal batch sizes for new items and for recovery. Marshall and Archibald [20] proposed a EPQ model with cycles consisting of multiple production runs followed by multiple recovery runs, where recovery converts returned items to either a serviceable level or into components that can be processed into serviceable items during the production runs. This reduces waste from disposing returned items that are not directly recoverable, which was the case in earlier models.

The authors of the above works assumed a constant demand rate. Alamri [21] broke from this trend by deriving a global optimal solution to a production-repair inventory model running a $(1,1)$ policy, where the demand, return, production, repair, and deterioration rates are arbitrary time-varying functions. Sun et al. [22] proposed a $(P, R)$-policy model in which the demand function is a trapezoidal type function of time that describes the introduction, maturity, and decline phases of a product's full life cycle. However, the rates of production and repair are constant. 
In this paper, we extend the EPQ model in [20] to the case of functions of time for the demand, production, and recovery (or repair) rates. The returned items are recovered through two channels - by repairing the returned items to a serviceable level or by converting the returned items into raw material that can be processed into serviceable items. The criterion for repair or conversion is the quality level of the returned items, where we assume that a returned item possesses one of two quality levels-reparable or not reparable but can be converted into raw material. However, for simplicity, we consider a $(1,1)$ policy instead of a $(P, R)$ policy. We also assume that recovery is less than demand, hence any surplus demand is satisfied by producing serviceable items from raw material that are procured from external sources in a single lot. We propose a unit time total cost expression and a numerical solution procedure to find the optimal recovery batch size. We illustrate our solution procedure with a numerical example where the rates are exponentially increasing functions of time. We perform numerical sensitivity analysis to observe how changes in the return portion, recovery portion, and unit repair cost affect the optimal policy.

This paper is organized as follows: Section 2 details the mathematical formulation of the propose model and the unit time total cost expression. Section 3 discusses a numerical solution procedure that can be used to find the optimal recovery batch size. Section 4 illustrates the solution procedure with an example and provides managerial insights through three numerical sensitivity analyses. Finally, Section 5 concludes the paper.

\section{Mathematical formulation}

The diagram in Fig. 1 shows the flow of items in the proposed inventory system. Raw material is supplied by external procurement and by converting irreparable returned items, which is then processed into serviceable items to satisfy demand. On the other hand, returned items are accumulated and divided into two groups. The first group is in a condition such that the items can be repaired to a serviceable level to satisfy demand. The second group is irreparable but is in a condition such that the items can be converted to raw material that can be processed into serviceable items.

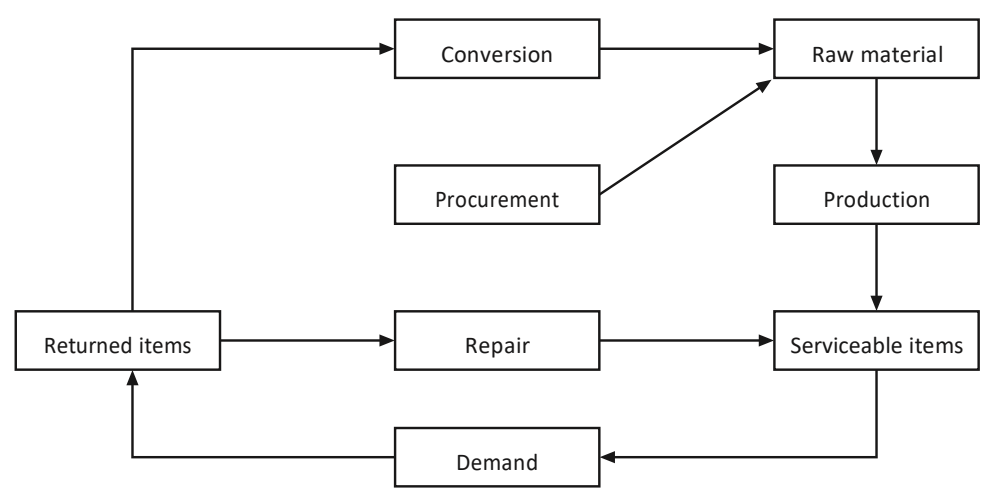

Fig. 1. Flow of items in the proposed inventory system.

In order to formulate the mathematical model of the proposed inventory system, the following assumptions and notations are used:

1. A single-item inventory system is considered over an infinite planning horizon.

2. The demand rate is a known function of time and is denoted by $D(t)$. 
3. The demand for serviceable items is satisfied by repairing returned items and by producing from raw material. The repair rate and production rate are known functions of time and are denoted by $R(t)$ and $P(t)$, respectively, where $R(t), P(t)>D(t)$.

4. The return rate is $\theta D(t)$, where $\theta$ is a constant and $0<\theta<1$.

5. A portion $\alpha$ of the returned items is in a condition such that they can repaired to an as good as new condition. The remaining portion $(1-\alpha)$ is in a condition such that they cannot be repaired but can be converted to raw material for the production process. The conversion rate is a known function of time and is denoted by $C(t)$, where $C(t)>D(t)$.

6. The raw material supply is further topped up by an external procurement that has an infinite rate of replenishment.

7. Each inventory cycle has one repair run and one production run.

8. Shortages are not allowed.

9. An incentive is given to the manufacturer in the form of a rebate for each unit of returned item that is successfully reused.

10. The following costs are considered:
- $K$,
total setup cost
- $c_{p}$,
unit holding cost of serviceable items per unit time
- $c_{r}$, unit holding cost of returned items per unit time
- $c_{m}$,
- $s_{p}$, unit holding cost of raw material per unit time
- $s_{r}$, unit production cost
- $s_{c}$ unit repair cost
- $s_{m}$, unit conversion cost
- $r$ unit raw material cost unit rebate for reuse

An example of the movement of inventory level of the proposed inventory system is illustrated in Fig. 2 for increasing $D(t), P(t), R(t)$, and $C(t)$. Let $I_{p}(t)$ denote the inventory level of the serviceable items at time $t, I_{r}(t)$ the inventory level of the returned items at time $t$, and $I_{m}(t)$ the inventory level of the raw material at time $t$. The inventory cycle starts running at time $T_{0}$, during which the repair run begins, which increases $I_{p}(t)$ at the rate $R(t)$ $-D(t)$ until time $T_{1}$, during which the repair run ends. Then $I_{p}(t)$ drops due to demand at the rate $D(t)$ until time $T_{3}$, during which it drops to 0 . For ease of reference, we shall refer to the period $\left[T_{0}, T_{1}\right]$ as the repair uptime period, $\left[T_{1}, T_{3}\right]$ as the repair downtime period, and $\left[T_{0}, T_{3}\right]$ as the repair period.

The cycle's production run starts at time $T_{3}$, which increases $I_{p}(t)$ at the rate $P(t)-D(t)$ until time $T_{4}$, during which the production run ends. Then $I_{p}(t)$ drops due to demand at the rate $D(t)$ until time $T_{5}$, during which it depletes to 0 . We shall refer to the period $\left[T_{3}, T_{4}\right]$ as the production uptime period, $\left[T_{4}, T_{5}\right]$ as the production downtime period, and $\left[T_{3}, T_{5}\right]$ as the production period.

The returned items that are in stock at the beginning of the cycle and the items that are returned during the repair uptime period are either repaired to a condition that is as good as new or converted to raw material for the production run. However, the returned items that are collected during the period $\left[T_{1}, T_{5}\right]$ are not reused in the current cycle. At time $T_{3}$, a shipment of raw material is received from external procurement, and together with the raw material that are obtained from converting returned items, these items are consumed by the production run at the rate $P(t)$ over the production uptime period. During the repair uptime period, the consumption of returned items by the repair run causes $I_{r}(t)$ to decrease at the rate $R(t)-\theta D(t)$. During the period $\left[T_{1}, T_{2}\right]$, the conversion process causes $I_{r}(t)$ to decrease at the rate $C(t)-\theta D(t) . I_{r}(t)$ does not decline to zero at time $T_{2}$ because the returned items that are collected during the period $\left[T_{1}, T_{2}\right]$ are not reused in the current cycle. Then $I_{r}(t)$ increases at the rate $\theta D(t)$ during the period $\left[T_{2}, T_{5}\right]$. 
Since a portion $\alpha$ of the returned items at the beginning of the cycle and the items that are returned during the period $\left[T_{0}, T_{1}\right]$ are repaired to meet demand during the period $\left[T_{0}\right.$, $T_{3}$ ], we have

$$
\alpha\left[I_{r}\left(T_{0}\right)+\int_{T_{0}}^{T_{1}} \theta D(t) d t\right]=\int_{T_{0}}^{T_{3}} D(t) d t=\int_{T_{0}}^{T_{1}} R(t) d t
$$

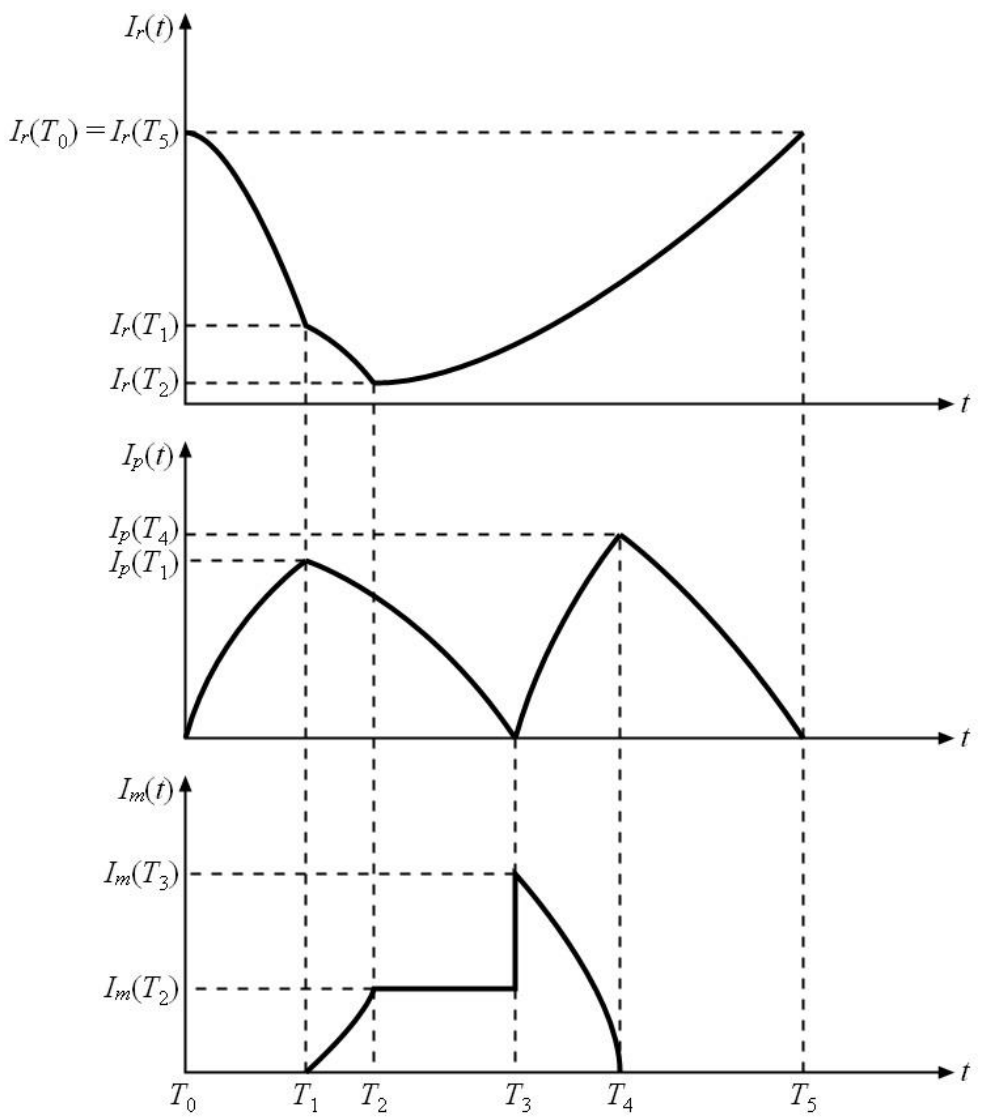

Fig. 2. Inventory movement the proposed inventory system for increasing rates.

We set $I_{r}\left(T_{0}\right)=I_{r}\left(T_{5}\right)$. Since $I_{r}\left(T_{5}\right)$ equals the number of returned items that are not reused in the current cycle, we have

which gives us

$$
I_{r}\left(T_{0}\right)=\int_{T_{1}}^{T_{5}} \theta D(t) d t
$$

$$
\alpha \int_{T_{0}}^{T_{5}} \theta D(t) d t=\int_{T_{0}}^{T_{1}} R(t) d t
$$

The remaining $(1-\alpha)$ portion of the returned items is converted to raw material. The conversion process occurs over the period $\left[T_{1}, T_{2}\right]$. We have

$$
(1-\alpha) \int_{T_{0}}^{T_{5}} \theta D(t) d t=\int_{T_{1}}^{T_{2}} C(t) d t
$$

Since $C(t)>D(t)$, to ensure that $T_{2}<T_{3}$, we propose the condition that the demand during the period $\left[T_{1}, T_{3}\right]$ is greater than the number of returned items that are converted to raw material. This condition can be written as 


$$
\alpha>\frac{1}{2}\left[1+\frac{\int_{T_{0}}^{T_{1}} D(t) d t}{\int_{T_{0}}^{T_{5}} \theta D(t) d t}\right]>\frac{1}{2}
$$

At time $T_{1}$, all reparable returned items are fully repaired, and the remaining units are awaiting conversion to raw material. Thus, we have

$$
I_{r}\left(T_{1}\right)=(1-\alpha) \int_{T_{0}}^{T_{5}} \theta D(t) d t .
$$

At time $T_{2}$, after the conversion process is completed, the inventory level of returned items equals the amount that is returned during the period $\left[T_{1}, T_{2}\right]$ that is unused. Thus, we have

$$
I_{r}\left(T_{2}\right)=\int_{T_{1}}^{T_{2}} \theta D(t) d t .
$$

After the raw material supply is topped up at time $T_{3}$, which is necessary if $\theta<1$, these units are processed into serviceable items by the production run to meet demand during the production period. Thus, we have

$$
I_{m}\left(T_{3}\right)=\int_{T_{3}}^{T_{5}} D(t) d t
$$

The movements of the inventory levels are described by the following boundary value problems:

$$
\begin{aligned}
& \frac{d I_{p}(t)}{d t}=R(t)-D(t), \quad t \in\left[T_{0}, T_{1}\right], \quad I_{p}\left(T_{0}\right)=0, \\
& \frac{d I_{p}(t)}{d t}=-D(t), \quad t \in\left[T_{1}, T_{3}\right], \quad I_{p}\left(T_{3}\right)=0, \\
& \frac{d I_{p}(t)}{d t}=P(t)-D(t), \quad t \in\left[T_{3}, T_{4}\right], \quad I_{p}\left(T_{3}\right)=0, \\
& \frac{d I_{p}(t)}{d t}=-D(t), \quad t \in\left[T_{4}, T_{5}\right], \quad I_{p}\left(T_{5}\right)=0, \\
& \frac{d I_{r}(t)}{d t}=-R(t)+\theta D(t), \quad t \in\left[T_{0}, T_{1}\right], \quad I_{r}\left(T_{1}\right)=(1-\alpha) \int_{T_{0}}^{T_{5}} \theta D(t) d t, \\
& \frac{d I_{r}(t)}{d t}=-C(t)+\theta D(t), \quad t \in\left[T_{1}, T_{2}\right], \quad I_{r}\left(T_{2}\right)=\int_{T_{1}}^{T_{2}} \theta D(t) d t, \\
& \frac{d I_{r}(t)}{d t}=\theta D(t), \quad t \in\left[T_{2}, T_{5}\right], \quad I_{r}\left(T_{2}\right)=\int_{T_{1}}^{T_{2}} \theta D(t) d t, \\
& \frac{d I_{m}(t)}{d t}=C(t), \quad t \in\left[T_{1}, T_{2}\right], \quad I_{m}\left(T_{1}\right)=0, \\
& \frac{d I_{m}(t)}{d t}=0, \quad t \in\left[T_{2}, T_{3}\right], \quad I_{m}\left(T_{3}\right)=\int_{T_{3}}^{T_{5}} D(t) d t, \\
& \frac{d I_{m}(t)}{d t}=-P(t), \quad t \in\left[T_{3}, T_{4}\right], \quad I_{m}\left(T_{4}\right)=0 .
\end{aligned}
$$

The solutions to (1)-(10) are, respectively, given by

$$
\begin{aligned}
& I_{p}(t)=\int_{T_{0}}^{t}[R(w)-D(w)] d w, \quad t \in\left[T_{0}, T_{1}\right], \\
& I_{p}(t)=\int_{t}^{T_{3}} D(w) d w, \quad t \in\left[T_{1}, T_{3}\right]
\end{aligned}
$$




$$
\begin{aligned}
& I_{p}(t)=\int_{T_{3}}^{t}[P(w)-D(w)] d w, \quad t \in\left[T_{3}, T_{4}\right], \\
& I_{p}(t)=\int_{t}^{T_{5}} D(w) d w, \quad t \in\left[T_{4}, T_{5}\right], \\
& I_{r}(t)=\int_{t}^{T_{1}}[R(w)-\theta D(w)] d w+(1-\alpha) \int_{T_{0}}^{T_{5}} \theta D(w) d w, \quad t \in\left[T_{0}, T_{1}\right], \\
& I_{r}(t)=\int_{t}^{T_{2}}[C(w)-\theta D(w)] d w+\int_{T_{1}}^{T_{2}} \theta D(w) d w, \quad t \in\left[T_{1}, T_{2}\right], \\
& I_{r}(t)=\int_{T_{1}}^{t} \theta D(w) d w, \quad t \in\left[T_{2}, T_{5}\right], \\
& I_{m}(t)=\int_{T_{1}}^{t} C(w) d w, \quad t \in\left[T_{1}, T_{2}\right], \\
& I_{m}(t)=(1-\alpha) \int_{T_{0}}^{T_{5}} \theta D(w) d w, \quad \in\left[T_{2}, T_{3}\right], \\
& I_{m}(t)=\int_{t}^{T_{4}} P(w) d w, \quad t \in\left[T_{3}, T_{4}\right] .
\end{aligned}
$$

Let $I_{x}(a, b)(x=p, r, m)$ denote the area under the graphs in Fig. 2 during the period $t \in$ $[a, b]$. Then from $(11)-(20)$, we have

$$
\begin{aligned}
& I_{p}\left(T_{0}, T_{1}\right)=\int_{T_{0}}^{T_{1}}\left(\int_{T_{0}}^{t}[R(w)-D(w)] d w\right) d t \\
& I_{p}\left(T_{1}, T_{3}\right)=\int_{T_{1}}^{T_{3}}\left(\int_{t}^{T_{3}} D(w) d w\right) d t \\
& I_{p}\left(T_{3}, T_{4}\right)=\int_{T_{3}}^{T_{4}}\left(\int_{T_{3}}^{t}[P(w)-D(w)] d w\right) d t \\
& I_{p}\left(T_{4}, T_{5}\right)=\int_{T_{4}}^{T_{5}}\left(\int_{t}^{T_{5}} D(w) d w\right) d t \\
& I_{r}\left(T_{0}, T_{1}\right)=\int_{T_{0}}^{T_{1}}\left(\int_{t}^{T_{1}}[R(w)-\theta D(w)] d w+[1-\alpha] \int_{T_{0}}^{T_{5}} \theta D(w) d w\right) d t, \\
& I_{r}\left(T_{1}, T_{2}\right)=\int_{T_{1}}^{T_{2}}\left(\int_{t}^{T_{2}}[C(w)-\theta D(w)] d w+\int_{T_{1}}^{T_{2}} \theta D(w) d w\right) d t \\
& I_{r}\left(T_{2}, T_{5}\right)=\int_{T_{2}}^{T_{5}}\left(\int_{T_{1}}^{t} \theta D(w) d w\right) d t \\
& I_{m}\left(T_{1}, T_{2}\right)=\int_{T_{1}}^{T_{2}}\left(\int_{T_{1}}^{t} C(w) d w\right) d t \\
& I_{m}\left(T_{2}, T_{3}\right)=\int_{T_{2}}^{T_{3}}\left([1-\alpha] \int_{T_{0}}^{T_{5}} \theta D(w) d w\right) d t \\
& I_{m}\left(T_{3}, T_{4}\right)=\int_{T_{3}}^{T_{4}}\left(\int_{t}^{T_{4}} P(w) d w\right) d t .
\end{aligned}
$$

Reducing (21)-(30) using integration by parts yields 


$$
\begin{aligned}
& I_{p}\left(T_{0}, T_{1}\right)=\int_{T_{0}}^{T_{1}}\left(T_{1}-t\right)[R(t)-D(t)] d t \\
& I_{p}\left(T_{1}, T_{3}\right)=\int_{T_{1}}^{T_{3}}\left(t-T_{1}\right) D(t) d t \\
& I_{p}\left(T_{3}, T_{4}\right)=\int_{T_{3}}^{T_{4}}\left(T_{4}-t\right)[P(t)-D(t)] d t \\
& I_{p}\left(T_{4}, T_{5}\right)=\int_{T_{4}}^{T_{5}}\left(t-T_{4}\right) D(t) d t \\
& I_{r}\left(T_{0}, T_{1}\right)=\int_{T_{0}}^{T_{1}}\left(t-T_{0}\right)[R(t)-\theta D(t)] d t+\left(T_{1}-T_{0}\right)(1-\alpha) \int_{T_{0}}^{T_{5}} \theta D(t) d t, \\
& I_{r}\left(T_{1}, T_{2}\right)=\int_{T_{1}}^{T_{2}}\left(t-T_{1}\right)[C(t)-\theta D(t)] d t+\left(T_{2}-T_{1}\right) \int_{T_{1}}^{T_{2}} \theta D(t) d t \\
& I_{r}\left(T_{2}, T_{5}\right)=T_{5} \int_{T_{1}}^{T_{5}} \theta D(t) d t-T_{2} \int_{T_{1}}^{T_{2}} \theta D(t) d t-\int_{T_{2}}^{T_{5}} t \theta D(t) d t \\
& I_{m}\left(T_{1}, T_{2}\right)=\int_{T_{1}}^{T_{2}}\left(T_{2}-t\right) C(t) d t \\
& I_{m}\left(T_{2}, T_{3}\right)=\left(T_{3}-T_{2}\right)(1-\alpha) \int_{T_{0}}^{T_{5}} \theta D(t) d t, \\
& I_{m}\left(T_{3}, T_{4}\right)=\int_{T_{3}}^{T_{4}}\left(t-T_{3}\right) P(t) d t .
\end{aligned}
$$

Without loss of generality, we shall set $T_{0}=0$. Then the unit time total cost, TCUT, is given by

$$
\begin{aligned}
\operatorname{TCUT}\left(T_{1}, T_{2}, T_{3},\right. & \left.T_{4}, T_{5}\right) \\
& =\frac{1}{T_{5}}\left\{K+c_{p}\left[I_{p}\left(0, T_{1}\right)+I_{p}\left(T_{1}, T_{3}\right)+I_{p}\left(T_{3}, T_{4}\right)+I_{p}\left(T_{4}, T_{5}\right)\right]\right. \\
& +c_{r}\left[I_{r}\left(0, T_{1}\right)+I_{r}\left(T_{1}, T_{2}\right)+I_{r}\left(T_{2}, T_{5}\right)\right] \\
& +c_{m}\left[I_{m}\left(T_{1}, T_{2}\right)+I_{m}\left(T_{2}, T_{3}\right)+I_{m}\left(T_{3}, T_{4}\right)\right] \\
& +\left[s_{r} \alpha+s_{c}(1-\alpha)-r\right] \int_{0}^{T_{5}} \theta D(t) d t+s_{p} \int_{T_{3}}^{T_{5}} D(t) d t \\
& \left.+s_{m} \int_{0}^{T_{5}}(1-\theta) D(t) d t\right\} .
\end{aligned}
$$

The problem is to minimize $\operatorname{TCUT}\left(T_{1}, T_{2}, T_{3}, T_{4}, T_{5}\right)$ subject to the constraints

$$
\begin{aligned}
& \int_{0}^{T_{1}} R(t) d t=\int_{0}^{T_{3}} D(t) d t \\
& \int_{T_{3}}^{T_{4}} P(t) d t=\int_{T_{3}}^{T_{5}} D(t) d t \\
& \int_{0}^{T_{1}} R(t) d t=\alpha \int_{0}^{T_{5}} \theta D(t) d t \\
& \int_{T_{1}}^{T_{2}} C(t) d t=(1-\alpha) \int_{0}^{T_{5}} \theta D(t) d t
\end{aligned}
$$




$$
\alpha>\frac{1}{2}\left[1+\frac{\int_{0}^{T_{1}} D(t) d t}{\int_{0}^{T_{5}} \theta D(t) d t}\right],
$$

where (32) and (33) ensure that all demands are satisfied by either repair or production, (34) ensures that the portion $\alpha$ of the returned items is repaired, (35) ensures that the portion $(1-\alpha)$ of the returned items is converted to raw materials, and (36) ensures that $T_{2}<T_{3}$. Moreover, these constraints ensure that

if $0<\theta<1$.

$$
0<T_{1}<T_{2}<T_{3}<T_{4}<T_{5}
$$

\section{Solution procedure}

Let $Q$ denote the number of returned items that enters the system in the period $\left[0, T_{5}\right]$. Then $Q$ is given by

$$
Q=\int_{0}^{T_{5}} \theta D(t) d t
$$

Since it is clear from (37) that $T_{5}$ is a function of $Q$, we shall write

$$
T_{5}=f_{5}(Q) \text {. }
$$

From (34), $T_{1}$ is a function of $T_{5}$, and hence of $Q$, and thus we shall write

$$
T_{1}=f_{1}(Q) \text {. }
$$

From (35), $T_{2}$ is a function of $T_{1}$ and $T_{5}$, and hence of $Q$, and thus we shall write

$$
T_{2}=f_{2}(Q) \text {. }
$$

Similarly, from (32) and (33), both $T_{3}$ and $T_{4}$ are functions of $Q$, and thus we shall write

$$
\begin{aligned}
& T_{3}=f_{3}(Q), \\
& T_{4}=f_{4}(Q) .
\end{aligned}
$$

After substituting (38)-(42) into (31), the constrained problem is converted into the following unconstrained problem:

$$
\begin{aligned}
\operatorname{Min} T C U T(Q) & =\frac{1}{f_{5}}\left\{K+c_{p}\left[\int_{0}^{f_{5}} t D(t) d t-\int_{0}^{f_{1}} t R(t) d t-\int_{f_{3}}^{f_{4}} t P(t) d t\right]\right. \\
& +c_{r}\left[\int_{0}^{f_{1}} t[R(t)-\theta D(t)] d t+\int_{f_{1}}^{f_{2}} t[C(t)-\theta D(t)] d t\right. \\
& \left.+f_{5} \int_{f_{1}}^{f_{5}} \theta D(t) d t-\int_{f_{2}}^{f_{5}} t \theta D(t) d t\right] \\
& +c_{m}\left[\int_{f_{3}}^{f_{4}}\left(t-f_{3}\right) P(t) d t+\int_{f_{1}}^{f_{2}}\left(f_{3}-t\right) C(t) d t\right] \\
& \left.+S \int_{0}^{f_{5}} \theta D(t) d t+s_{p} \int_{f_{3}}^{f_{5}} D(t) d t+s_{m} \int_{0}^{f_{5}}(1-\theta) D(t) d t\right\}
\end{aligned}
$$

where $S=s_{r} \alpha+s_{c}(1-\alpha)-r$. Note that $f_{k}(Q)(k=1,2,3,4,5)$ doesn't need to be closed, since whenever necessary, the desired value can be found numerically. The necessary condition to solve the above problem is

Let

$$
\frac{d(T C U T)}{d Q}=0 \text {. }
$$

$$
T C U T=\frac{w}{f_{5}^{\prime}}
$$

where $w$ is the expression inside the curly braces in (43), so that (44) gives the optimality condition 


$$
f_{5} w^{\prime}-w f_{5}^{\prime}=0
$$

Differentiating $w$ with respect to $Q$ gives

$$
\begin{aligned}
& w^{\prime}=c_{p}\left[f_{5}^{\prime} f_{5} D\left(f_{5}\right)-f_{1}^{\prime} f_{1} R\left(f_{1}\right)-f_{4}^{\prime} f_{4} P\left(f_{4}\right)+f_{3}^{\prime} f_{3} P\left(f_{3}\right)\right] \\
& +c_{r}\left[f_{1}^{\prime} f_{1} R\left(f_{1}\right)+f_{2}^{\prime} f_{2} C\left(f_{2}\right)-f_{1}^{\prime} f_{1} C\left(f_{1}\right)-f_{1}^{\prime} f_{5} \theta D\left(f_{1}\right)+f_{5}^{\prime} \int_{f_{1}}^{f_{5}} \theta D(t) d t\right] \\
& +c_{m}\left[f_{4}^{\prime}\left(f_{4}-f_{3}\right) P\left(f_{4}\right)+f_{2}^{\prime}\left(f_{3}-f_{2}\right) C\left(f_{2}\right)-f_{1}^{\prime}\left(f_{3}-f_{1}\right) C\left(f_{1}\right)\right. \\
& \left.-f_{3}^{\prime} \int_{f_{3}}^{f_{4}} P(t) d t+f_{3}^{\prime} \int_{f_{1}}^{f_{2}} C(t) d t\right]+\left(S \theta+s_{m}-s_{m} \theta\right) f_{5}^{\prime} D\left(f_{5}\right) \\
& +s_{p}\left[f_{5}^{\prime} D\left(f_{5}\right)-f_{3}^{\prime} D\left(f_{3}\right)\right] .
\end{aligned}
$$

The following expressions for the derivatives $f_{1}{ }^{\prime}, f_{2}{ }^{\prime}, f_{3}{ }^{\prime}, f_{4}{ }^{\prime}$, and $f_{5}{ }^{\prime}$ can be easily derived from (32)-(35) and (37):

$$
\begin{aligned}
& f_{1}^{\prime}=\frac{\alpha}{R\left(f_{1}\right)^{\prime}} \\
& f_{2}^{\prime}=\frac{\alpha C\left(f_{1}\right)+(1-\alpha) R\left(f_{1}\right)}{R\left(f_{1}\right) C\left(f_{2}\right)}, \\
& f_{3}^{\prime}=\frac{\alpha}{D\left(f_{3}\right)}, \\
& f_{4}^{\prime}=\left[\frac{1}{\theta}-\alpha+\frac{\alpha P\left(f_{3}\right)}{D\left(f_{3}\right)}\right] \frac{1}{P\left(f_{4}\right)^{\prime}} \\
& f_{5}^{\prime}=\frac{1}{\theta D\left(f_{5}\right)} .
\end{aligned}
$$

To determine the optimal value of $Q,(45)$ is solved. Then the optimal values of $T_{1}, T_{2}$, $T_{3}, T_{4}$, and $T_{5}$ are found from (38)-(42). Then the minimum TCUT is determined from the following equation:

$$
T C U T=w / f_{5}=w^{\prime} / f_{5}^{\prime} .
$$

\section{Numerical examples}

In this section, we illustrate the proposed model with four numerical examples. In the first example, we consider exponential functions of time for the demand, production, repair, and conversion rates. Using exponential functions enables us to derive closed-form expressions for $f_{k}(Q)(k=1,2,3,4,5)$. Using numerical sensitivity analysis in the second, third, and fourth examples, we observe how changes in the unit repair cost, the unit rebate for reuse, and the portion of reparable and non-reparable returned items affect the optimal TCUT for various $\theta$. These three factors are considered because they may affect how an inventory manager handles his reuse policy.

\subsection{Example 1: Exponential functions for the demand, production, repair, and conversion rates}

Suppose that the rates are given by following functions:

$$
D(t)=b e^{a t}, \quad P(t)=\phi_{p} e^{\pi_{p} t}, \quad R(t)=\phi_{r} e^{\pi_{r} t}, \quad C(t)=\phi_{c} e^{\pi_{c} t},
$$

where $b, \phi_{p}, \phi_{r}, \phi_{c}>0$ and $a, \pi_{p}, \pi_{r}, \pi_{c} \geq 0$.

From (37), we obtain

$$
f_{5}=\frac{1}{a} \ln \left(\frac{a Q}{b \theta}+1\right) .
$$


From (34), we obtain

From (35), we obtain

$$
f_{1}=\frac{1}{\pi_{r}} \ln \left(\frac{\alpha \pi_{r} Q}{\phi_{r}}+1\right) .
$$

From (32), we obtain

$$
f_{2}=\frac{1}{\pi_{c}} \ln \left[e^{\pi_{c} f_{1}}+\frac{(1-\alpha) \pi_{c} Q}{\phi_{c}}\right] \text {. }
$$

And from (33), we obtain

$$
f_{3}=\frac{1}{a} \ln \left[\frac{a \phi_{r}}{b \pi_{r}}\left(e^{\pi_{r} f_{1}}-1\right)+1\right] \text {. }
$$

$$
f_{4}=\frac{1}{\pi_{p}} \ln \left[e^{\pi_{p} f_{3}}+\frac{b \pi_{p}}{a \phi_{p}}\left(\frac{a Q}{b \theta}+1-e^{a f_{3}}\right)\right] .
$$

After some algebraic manipulation, we obtain

$$
\begin{aligned}
& w=K+\frac{\left(c_{r}-c_{p}\right) \phi_{r}}{\pi_{r}^{2}}\left[e^{\pi_{r} f_{1}}\left(\pi_{r} f_{1}-1\right)+1\right]+\frac{\left(c_{p}-\theta c_{r}\right) b}{a^{2}}\left[e^{a f_{5}}\left(a f_{5}-1\right)+1\right] \\
& +\frac{\left(c_{m}-c_{p}\right) \phi_{p}}{\pi_{p}^{2}}\left[e^{\pi_{p} f_{4}}\left(\pi_{p} f_{4}-1\right)-e^{\pi_{p} f_{3}}\left(\pi_{p} f_{3}-1\right)\right]+\frac{c_{r} f_{5} \theta b}{a}\left(e^{a f_{5}}-e^{a f_{1}}\right) \\
& +\frac{\left(c_{r}-c_{m}\right) \phi_{c}}{\pi_{c}^{2}}\left[e^{\pi_{c} f_{2}}\left(\pi_{c} f_{2}-1\right)-e^{\pi_{c} f_{1}}\left(\pi_{c} f_{1}-1\right)\right] \\
& +\left[\frac{S \theta+s_{m}(1-\theta)}{a}\right] b\left(e^{a f_{5}}-1\right) \\
& +c_{m} f_{3}\left[\frac{\phi_{c}}{\pi_{c}}\left(e^{\pi_{c} f_{2}}-e^{\pi_{c} f_{1}}\right)-\frac{\phi_{p}}{\pi_{p}}\left(e^{\pi_{p} f_{4}}-e^{\pi_{p} f_{3}}\right)\right]+\frac{s_{p} b}{a}\left(e^{a f_{5}}-e^{a f_{3}}\right)
\end{aligned}
$$

and

$$
\begin{aligned}
& w^{\prime}=c_{p}\left[\frac{f_{5}-f_{4}}{\theta}+\alpha\left(f_{4}-f_{1}\right)-\frac{\alpha \phi_{p}}{b} e^{\left(\pi_{p}-a\right) f_{3}}\left(f_{4}-f_{3}\right)\right] \\
& +c_{r}\left[\alpha f_{1}+(1-\alpha) f_{2}+\frac{\alpha \phi_{c}}{\phi_{r}} e^{\left(\pi_{c}-\pi_{r}\right) f_{1}}\left(f_{2}-f_{1}\right)+\frac{1}{a}\left(1-e^{a f_{1}-a f_{5}}\right)\right. \\
& \left.-\frac{\alpha \theta b f_{5}}{\phi_{r}} e^{\left(a-\pi_{r}\right) f_{1}}\right] \\
& +c_{m}\left[\left(\frac{1}{\theta}-\alpha\right)\left(f_{4}-f_{3}\right)+(1-\alpha)\left(f_{3}-f_{2}\right)-\frac{\alpha \phi_{c}}{\phi_{r}} e^{\left(\pi_{c}-\pi_{r}\right) f_{1}}\left(f_{2}-f_{1}\right)\right. \\
& \left.+\frac{\alpha}{b e^{a f_{3}}}\left\{\phi_{p} e^{\pi_{p} f_{3}}\left(f_{4}-f_{3}\right)-\frac{b}{a}\left(e^{a f_{5}}-e^{a f_{3}}\right)+\frac{\phi_{c}}{\pi_{c}}\left(e^{\pi_{c} f_{2}}-e^{\pi_{c} f_{1}}\right)\right\}\right] \\
& +S+\frac{s_{m}(1-\theta)}{\theta}+s_{p}\left(\frac{1}{\theta}-\alpha\right) .
\end{aligned}
$$

We illustrate the solution procedure with a numerical example that uses the parameter values in Table 1. In the absence of actual data, these parameter values are chosen randomly but we assume that the setup cost is greater than the unit holding costs. For the purpose of illustrating the solution procedure, the accuracy of these values is inconsequential. The solution is found by solving

$$
f_{5} w^{\prime}-w f_{5}^{\prime}=0
$$

numerically. The root_scalar() solver from Python's scipy.optimize library is used to solve the equation. The optimal return quantity, $Q^{*}$, is computed to be 218.13 units, where 174.5 units are repaired to a serviceable condition and 43.63 units are converted to raw material. In addition, 145.33 units are produced from external raw material. The repair period is $[0,2.87]$ and the production period is $[2.87,5.88]$. The repair uptime period is $[0$, 
2.15] and the production uptime period is [2.87, 4.44]. Moreover, the returned items conversion process spans the period $[2.15,2.61]$. Finally, the optimal unit time total cost is TCUT* $=7267.05$.

Table 1. Parameter values for Example 1.

\begin{tabular}{ccccccc}
\hline$K$ & $h_{p}$ & $h_{r}$ & $h_{m}$ & $r$ & $s_{p}$ & $s_{r}$ \\
\hline 6000 & 10 & $0.5 h_{p}$ & $0.5 h_{r}$ & 0 & 100 & $0.5 s_{p}$ \\
\hline$s_{c}$ & $s_{m}$ & $\theta$ & $\alpha$ & $\phi_{p}$ & $\pi_{p}$ & $\phi_{r}$ \\
\hline $0.5 s_{r}$ & $0.9 s_{c}$ & 0.6 & 0.8 & 100 & 0.05 & 80 \\
\hline$\pi_{r}$ & $\phi_{c}$ & $\pi_{c}$ & $b$ & $a$ & & \\
\hline 0.015 & 90 & 0.02 & 60 & 0.01 & & \\
\hline
\end{tabular}

\subsection{Example 2: How the unit repair cost affect the optimal policy}

In this example, we observe how changes in the unit repair cost, $s_{r}$, affect the optimal TCUT for various $\theta$. We set the parameter values to be the same as those in Example 1, except for $s_{r}$, which we set to be the values $\left\{0.5 s_{p}, 0.7 s_{p}, 0.9 s_{p}, 1.225 s_{p}, 1.4 s_{p}\right\}$, and $\theta$, which we set to be the values $\{0.1,0.2, \ldots, 0.9,1\}$. The results are shown in Fig. 3 .

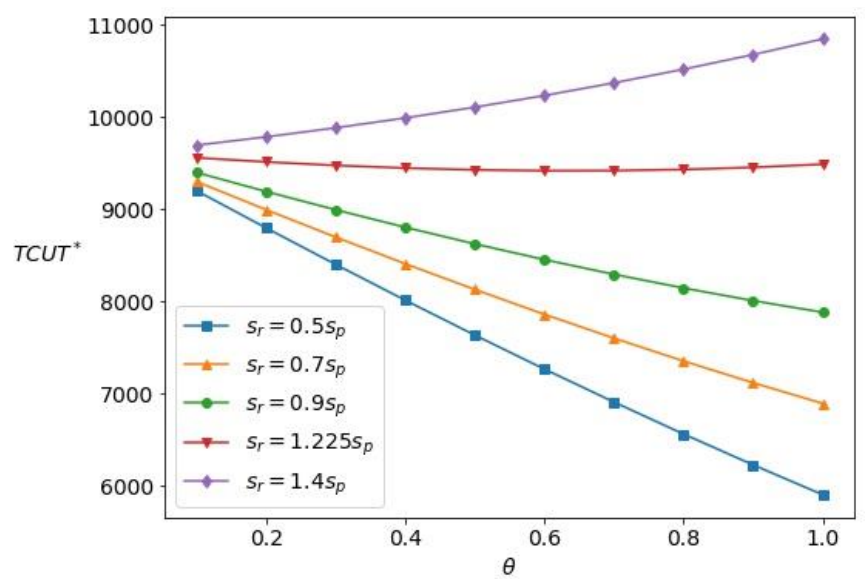

Fig. 3. Change of TCUT with respect to $\theta$ for various $s_{r}$.

It can be observed that the cases where $s_{r}<s_{p}$ favor a pure reuse policy $(\theta=1)$. The unit repair cost, $s_{r}$, includes the unit procurement cost of returned items. Hence the actual unit production cost must include the unit procurement cost of raw material as well, which gives us

Actual unit production cost $=s_{p}+s_{m}=1.225 s_{p}$.

The TCUT remains relatively stable for the case of $s_{r}=1.225 s_{p}$. However, the case where $s_{r}$ $=1.4 s_{p}$ favors a pure production policy $(\theta=0)$. This shows that an increase in $s_{r}$ will push the optimal policy from pure reuse to pure production. 


\subsection{Example 3: How the unit rebate for reuse affect the optimal policy}

In this example, we observe how changes in the unit rebate for reuse, $r$, which we set to zero in the two previous examples, affect the optimal TCUT for various $\theta$. We set the parameter values to be the same as those in Example 1, except for $s_{r}$, which we set to be $1.4 s_{p}, r$, which we set to be the values $\{0,10,20\}$, and $\theta$, which we set to be the values $\{0.1,0.2, \ldots, 0.9,1\}$. The results are shown in Fig. 4 .

It can be observed that when there is no rebate, a pure production policy is favored. However, a hybrid policy $(0<\theta<1)$ is favored when $r=10$, and when $r=20$, a pure reuse policy is favored. This supports the notion that a cost incentive can be given to offset a more expensive unit repair cost and push the optimal policy from pure production to pure reuse.

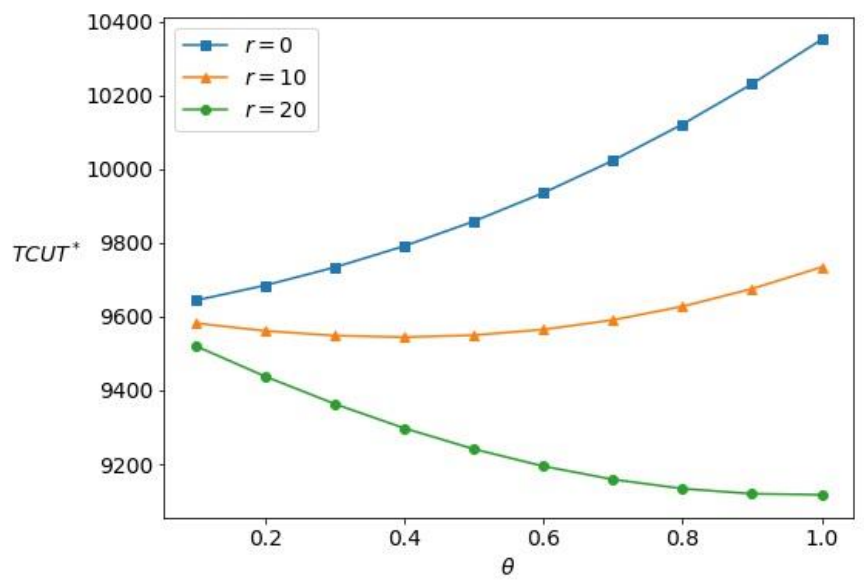

Fig. 4. Change of TCUT with respect to $\theta$ for various $r$.

\subsection{Example 4: How the portion of reparable returned items affect the optimal policy}

In this example, we observe how changes in the reparable portion of returned items, $\alpha$, affect the optimal TCUT for various $\theta$. We set the parameter values to be the same as those in Example 3, except for $r$, which we set to be 10, and $\theta$, which we set to be the values $\{0.1$, $0.2, \ldots, 0.9,1\}$. The results are shown in Fig. 5 .

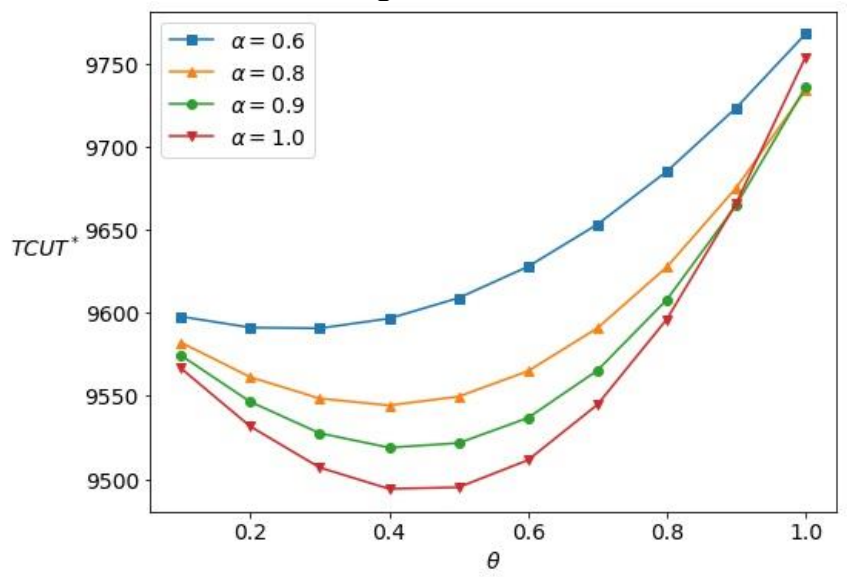

Fig. 5. Change of TCUT with respect to $\theta$ for various $\alpha$. 
It can be observed that when the rebate for reuse, $r$, is set to 10 , the optimal policy is a hybrid of production and repair rather than a pure policy. Despite setting the unit repair cost, $s_{r}$, to $1.4 s_{p}$, thus making repair more expensive than producing from raw material, the optimal TCUT decreases as the reparable portion $\alpha$ increases. However, the return portion, $\theta$, that gives the best TCUT, falls below 0.5 , which is not encouraging from a reuse point of view. As shown in Example 3, a higher $r$ is required to push the policy from low reuse to pure reuse.

\section{Conclusion}

In this paper, we proposed a mathematical model for an inventory system over an infinite planning horizon where a time-varying demand is satisfied by process cycles that consist of a production batch followed by a recovery batch. We considered three types of inventoryreturned items, serviceable items, and raw material. The returned items are recovered through two channels - recovery into serviceable items or recovery into raw materials. Serviceable items are acquired from two inputs - direct recovery from returned items or production from raw material. These raw materials are thus recovered from returned items and topped up through procurement from external sources whenever required. We proposed an expression for the unit time total cost and a numerical solution procedure to find the optimal policy.

Four numerical examples are presented. The first example illustrated the solution procedure for the case of exponentially increasing functions of time. The second example showed that increase in the unit repair cost pushes the optimal policy from pure repair to pure production. However, it was also shown that it is not necessary for repair to be cheaper than production, because an optimal hybrid policy can leverage the reduced inventory holding of serviceable items compared to a pure production policy.

The third example showed that in the case of higher unit repair costs, an appropriate rebate given for reuse can re-establish the optimality of hybrid or even pure repair policies. Realistically, such a rebate will have to come from neutral third parties such as the government, because it is an extra cost to be shouldered in order to force a non-optimal repair policy to become optimal. Alternatively, the stakeholders can choose to invest in improving technology to bring down the unit repair cost, while bearing with pure production policies in the meantime. From our model, we should be able to estimate how much rebate or how low the unit repair cost needs to be in order to produce an optimal hybrid policy.

Finally, the last example showed that direct recovery into serviceable items is preferred over recovery into raw material, but for higher unit repair costs, the optimal hybrid policy has a lower reuse rate $(\theta<0.5)$. This can be mitigated by giving a higher rebate $r$. Realistically, obtaining returned items at a directly-recoverable quality level is always a challenge; thus one can also think of the rebate as some sort of compensation so that more premium prices can be offered by the inventory manager for the procurement of higher quality returned items, and so that the end-users are encouraged to maintain the quality of the items they plan to return.

Two possible avenues for further research are immediately obvious. The first is the extension of the $(1,1)$ policy to the $(P, R)$ policy. The second is to compare the policy of direct recovery and conversion to raw material to a waste disposal policy where the nonreparable returned items are discarded at a cost. 


\section{References}

1. M. Fleischmann, J.M. Bloemhof-Ruwaard, R. Dekker, R. van der Laan, J.A. van Nunen, L.N. van Wassenhove, Eur. J. Op. Res. 103(1), 1 - 17 (1997)

2. T. Spengler, H. Püchert, T. Penkuhn, O. Rentz, Eur. J. Op. Res. 97(2), $308-326$ (1997)

3. F.W. Harris, Factory, The Magazine of Management 10(2), 135 - 136, 152 (1913)

4. D.A. Schrady, Nav. Res. Logist. 14(3), 391 - 398 (1967)

5. N. Nahmias, H. Rivera, Int. J. Prod. Res. 17(3), 215 - 221 (1979)

6. M.C. Mabini, L.M. Pintelon, L.F. Gelders, Int. J. Prod. Econ. 28(1), 21 - 33 (1992)

7. S.G. Koh, H. Hwang, K.I. Sohn, C.S. Ko, Comp. \& Ind. Eng. 43(1-2), 59 - 73 (2002)

8. I. Konstantaras, S. Papachristos, Comp. \& Ind. Eng. 55(3), 729 - 734 (2008)

9. R.H. Teunter, Comp. \& Ind. Eng. 46(3), 431 - 441 (2004)

10. I. Konstantaras, S. Papachristos, Int. J. Prod. Econ. 111(2), 707 - 712 (2008)

11. H.M. Wee, G.A. Widyadana, Comput. Math. with Appl. 59(8), 2933 - 2939 (2010)

12. K. Richter, Eur. J. Op. Res. 95(2), 313 - 324 (1996)

13. K. Richter, Int. J. Prod. Econ. 45(1-3), 443 - 448 (1996)

14. K. Richter, I. Dobos, Int. J. Prod. Econ. 59(1-3), 463 - 467 (1999)

15. I. Dobos, K. Richter, Cen. Eur. J. Op. Res. 8(2), 173 - 194 (2000)

16. R.H. Teunter, Nav. Res. Logist. 48(6), $484-495$ (2001)

17. I. Dobos, K. Richter, Cen. Eur. J. Op. Res. 11(1), 35 - 46 (2003)

18. I. Dobos, K. Richter, Int. J. Prod. Econ. 90(3), 311 - 323 (2004)

19. H. Mokhtari, Sustain. Prod. Consum. 15, 163 - 172 (2018)

20. S.E. Marshall, T.W. Archibald, Comp. \& Ind. Eng. 123, 134 - 147 (2018)

21. A.A. Alamri, Comp. \& Ind. Eng. 60(2), 236 - 247 (2011)

22. H. Sun, X. Xia, B. Liu, Comp. \& Ind. Eng. 142, https://doi.org/10.1016/j.cie.2020.106340 (2020) 